\title{
Screened bulk electron-phonon interactions in quantum-well wires
}

\author{
B. Tanatar \\ Department of Physics, Bilkent University, Bilkent, 06533 Ankara, Turkey
}

(Received 26 May 1993)

\begin{abstract}
We study the effects of dielectric screening on the scattering and relaxation rates due to electronphonon interactions in quasi-one-dimensional quantum-well wires. Interaction of confined electrons with bulk polar-optical and acoustic phonons are considered. Acoustic phonons are examined both in the deformation potential and the piezoelectric couplings. Screening effects are included via a temperature dependent dielectric function $\varepsilon_{T}(q)$ in the random-phase approximation, by renormalizing the electron-phonon interaction. We report numerical results for the GaAs system in the size-quantum-limit, where the lowering of screened electron-phonon scattering rates is found. The effects of screening are most significant for the electron-optical-phonon interaction. Mobility calculations using the screened electron-phonon interactions are also considered.
\end{abstract}

\section{INTRODUCTION}

Recent developments in molecular-beam-epitaxy (MBE) technology have made possible the fabrication of electronic structures based on the confinement of electrons in an essentially one-dimensional (1D) semiconductor, where the characteristic dimensions of the system are of the order of the de Broglie wavelength of the charge carriers. In these synthetic materials, the electron gas is quantized in two transverse directions, so the charge carriers can only move in the longitudinal direction. Since the electron gas has a small but finite extent in the confined directions compared with the axis of free motion, such structures are also named quasi-onedimensional (Q1D) systems. Owing to the limited number of available final states during the scattering process, the mobility of such systems is considerably enhanced, making them potentially important for high-speed device applications. Since their early prediction by Sakaki ${ }^{1}$ and subsequent fabrication, ${ }^{2-5}$ there has been a lot of interest in the transport properties of Q1D systems. Scattering mechanisms due to various processes have been examined both theoretically and experimentally. ${ }^{6-11} \mathrm{~A}$ transport theory in 1D or Q1D semiconductor structures has been developed for scattering mechanisms such as impurity, ${ }^{12}$ acoustic-phonon, ${ }^{6,13}$ and optical-phonon ${ }^{7,8,13}$ interactions. In a recent review by Bastard, Brum, and Ferreira, ${ }^{14}$ many properties of $1 \mathrm{D}$ semiconductor systems are discussed in detail.

To the best of our knowledge, the effects of the electron-acoustic-phonon scattering in quantum-well wires were first considered by Arora. ${ }^{6}$ Riddock and Ridley $^{7}$ and Leburton ${ }^{8}$ studied the electron-opticalphonon interaction in an infinite quantum-well model, and found that the $1 \mathrm{D}$ emission rates are less than the corresponding $3 \mathrm{D}$ rates. The size dependence of scattering rates in these $1 \mathrm{D}$ semiconductor materials was also reported by Leburton. ${ }^{8}$ Constantinou and Ridley ${ }^{15}$ considered a finite quantum well and calculated the electron-polar-optical-phonon scattering rates within the effective-mass approximation for a Q1D cylindrical quantum-well wire. They found that the scattering rates were lowered compared to the infinite confining potential approximation.

Electron-phonon scattering rates for 1D and Q1D electronic systems have also been calculated through Monte Carlo simulations. ${ }^{9,16,17}$ The low dimensional nature of these structures has rendered possible the inclusion of many subbands and more realistic confining potentials in such approaches. Simulations of optical- and acousticphonon scattering in Q1D systems with many subbands were performed for the size and confining potential-well height dependence. Calculations taking the many-body effects into account self-consistently, as well as polaron scattering rates $^{11}$ and polaronic energy ${ }^{18}$ in Q1D structures, have also been reported.

The scattering rates and mobilities for rectangular Q1D systems due to acoustic phonons coupled through piezoelectric interaction were also studied. ${ }^{19,20}$ The general conclusion of such investigations is that the piezoelectric scattering-limited mobilities in $\mathrm{GaAs}, \mathrm{InSb}$, and InAs are smaller than the acoustic-phonon (deformationpotential-coupling) limited mobility, except for strongly piezoelectric materials such as $\mathrm{CdS}$ and $\mathrm{ZnO}$, where piezoelectric scattering is dominant.

Mobility measurements and calculations in semiconductor structures have always been very important in determining the various scattering mechanisms within such systems. The hope of achieving very high mobilities by confining even more electrons compared with the Q2D heterojunctions has been the main motivation of the study of Q1D electron systems. A detailed and comparative study on phonon-limited mobility in Q1D semiconductors was given by Fishman, ${ }^{13}$ in which a simple model for the envelope wave function is employed. Similar calculations of mobility for rectangular cross-sectional quantum-well wires were reported by Lee and Vassell. ${ }^{20}$

We note that the transport relaxation time $\tau_{t}$ and the single-particle lifetime $\tau_{s}$ are in general different quantities. The relaxation time $\tau_{t}$ is what enters the mobility calculations, whereas the single-particle lifetime or 
the scattering time is related to the self-energy $\left[\tau_{s}^{-1} \sim\right.$ $\left.\operatorname{Im} \Sigma\left(k, \epsilon_{k}\right)\right]$. In effect, single-particle lifetime measures the time between the scattering events, and momentum relaxation time is weighted by a factor that depends on the angle of scattering. Only when the scattering potential is a $\delta$ function do we have $\tau_{t}=\tau_{s}$, and both transport and single-particle properties are characterized by the same quantity.

Screening effects have long been recognized to reduce the effects of electron-phonon interaction in semiconductor structures in various dimensions. In the usual treatment of scattering or relaxation time calculations, these screening effects have been incorporated by considering the Thomas-Fermi or Debye screening lengths in the appropriate electron-phonon interaction. We note that for the Thomas-Fermi screening approach to be valid, the wave vectors of interest must satisfy $k \ll k_{F}$, where $k_{F}$ is the Fermi wave vector. In the case of electronoptical-phonon interaction, the wave vectors in which $k \sim k_{0}$ are relevant, where $k_{0}=\left(2 m \omega_{0} / \hbar^{2}\right)^{1 / 2}$ and $\omega_{0}$ is the optical phonon frequency. Consequently, the Thomas-Fermi static screening of electron-phonon interaction is expected to be applicable if $k_{0} \ll k_{F}$. For GaAs heterostructures this implies the condition $n>$ $10^{18} \mathrm{~cm}^{-3}$ on the electron density (which corresponds to $n \sim 10^{6} \mathrm{~cm}^{-1}$ for $1 \mathrm{D}$ semiconductors). This is much higher than the electron densities typically encountered in the experiments. ${ }^{2}$ Better approximations to screening, namely, taking the full wave vector dependence of the dielectric function $\varepsilon(q)$ into account, are rarely used. Thus, motivated partly by the work of Constantinou and Ridley, ${ }^{15}$ we investigate the possible reduction in the scattering rates of Q1D quantum-well wires due to the effects of screened electron-phonon interactions. We stress that the phonons considered in this study are $3 \mathrm{D}$ (bulk), and we neglect any discussion of confined phonon modes. Some recent applications of the effect of electronconfined-phonon interactions in quantum-well wires can be found elsewhere. ${ }^{21}$

In this paper, our aim is to study the scattering rates due to electron-phonon interactions in Q1D structures. More specifically, we examine the electronoptical-phonon and electron-acoustic-phonon interactions. Acoustic phonons are considered in both the deformation potential and the piezoelectric couplings. We then investigate the effects of screened electron-phonon interactions on these phonon-limited scattering rates. Screening is introduced through the dielectric function $\varepsilon(q)$ for which we employ the random-phase approxima- tion (RPA). The dielectric response function for a Q1D semiconducting system has been calculated by Lee and Spector. ${ }^{22}$ Screening effects via a temperature dependent $1 \mathrm{D}$ dielectric function $\varepsilon_{T}(q)$ on the phonon-limited mobility were considered by Fishman. ${ }^{13}$ We also investigate the effects of screened electron-phonon interactions on the mobility, paying particular attention to the carrier density and the temperature dependence of $\mu$. Recently, we have calculated the effects of screening on the electronphonon scattering rates, with a rather crude approximation to the dielectric function $\varepsilon(q) .^{23}$

The rest of this paper is organized as follows. In Sec. II we introduce the wave function and energy levels for electrons confined in a cylindrical quantum wire, derive expressions for scattering rates due to various electron-phonon interactions, and introduce the temperature dependent dielectric function $\varepsilon_{T}(q)$. In Sec. III we present our numerical results of the phonon emission and absorption rates for electron-optical-phonon scattering, electron-acoustic-phonon interaction with deformation potential coupling, and electron-acoustic-phonon interaction with piezoelectric coupling. We also discuss the implications of our calculations on the temperature dependent phonon-limited mobility $\mu$.

\section{THEORY}

\section{A. Model}

We consider a model of electron gas, quantized in two transverse directions, so that the charge carriers can only move in the longitudinal direction. We choose the cross section of the system to be circular with radius $R$, hence the quantum wire geometry becomes cylindrical. Calculations have also been performed for wire of rectangular cross sections, ${ }^{1,6,8}$ but the size of the cross section, rather than its shape, is found ${ }^{24}$ to be relevant in determining various properties. In the size quantum limit (SQL), the radius $R$ of the quantum-well wire is much smaller than the thermal de Broglie wavelength of the charge carriers, so that only the ground state (lowest) subband is populated. As will be discussed later, we choose the size parameters in this work such that the SQL is attained and the intersubband scattering that otherwise would be important $^{25}$ is completely neglected. In the model of an infinite potential well confining the charge carriers, the normalized effective-mass wave function is given by

$$
\psi(\rho, \phi, z)= \begin{cases}J_{n}\left(k_{n, l} \rho\right) e^{i n \phi} e^{i k_{z} z} /\left[\left(\pi R^{2}\right)^{1 / 2} J_{n+1}\left(k_{n, l} R\right)\right], & \rho \leq R \\ 0, & \rho>R\end{cases}
$$

with the corresponding energy levels

$$
E=\frac{\hbar^{2}}{2 m}\left(k_{z}^{2}+k_{n, l}^{2}\right)
$$

where $k_{n, l}=x_{n, l} / R$ and $x_{n, l}$ is the $l$ th root of the Bessel function of the first kind $J_{n}(x)$.

\section{B. Scattering and relaxation rates}

The single-particle scattering rates can be obtained by summing over the final states using

$$
\frac{1}{\tau_{s}^{\left\{\begin{array}{c}
e \\
a
\end{array}\right\}}\left(k_{z}\right)}=\sum_{k_{z}^{\prime}} W^{\left\{\begin{array}{l}
e \\
a
\end{array}\right\}}\left(k_{z}, k_{z}^{\prime}\right)
$$


where the transition probability $\left.W^{\left\{\begin{array}{l}e \\ a\end{array}\right.}\right\}\left(k_{z}, k_{z}^{\prime}\right)$ of an electron, which forms a state $k_{z}$ to $k_{z}^{\prime}$ by emitting or absorbing a phonon, is given by the Fermi golden rule

$$
\left.\left.W^{\left\{\begin{array}{l}
e \\
a
\end{array}\right.}\right\}\left(k_{z}, k_{z}^{\prime}\right)=\frac{2 \pi}{\hbar} \mid M^{\left\{\begin{array}{l}
e \\
a
\end{array}\right.}\right\}\left.\right|^{2} \delta\left(E\left(k_{z}^{\prime}\right)-E\left(k_{z}\right) \pm \hbar \omega_{\mathbf{q}}\right) .
$$

Transport relaxation time, on the other hand, is calculated from

$$
\frac{1}{\tau_{t}^{\left\{\begin{array}{c}
e \\
a
\end{array}\right\}}\left(k_{z}\right)}=\sum_{k_{z}^{\prime}} W^{\left\{\begin{array}{l}
e \\
a
\end{array}\right\}}\left(k_{z}, k_{z}^{\prime}\right)\left(1-\frac{k_{z}^{\prime}}{k_{z}}\right)
$$

in which the extra factor describes the angle dependence of the scattering. The electron-phonon matrix element corresponding to the annihilation and creation of one phonon (upper and lower signs, respectively) is

$$
M^{\left\{\begin{array}{l}
e \\
a
\end{array}\right\}}=\left\langle k_{z}^{\prime}, N_{\mathbf{q}} \pm 1\left|H_{e-\mathbf{p h}}\right| k_{z}, N_{\mathbf{q}}\right\rangle
$$

where $N_{\mathbf{q}}$ is the phonon occupation factor and the electron-phonon interaction can be written as

$$
H_{e-\mathrm{ph}}=\sum_{\mathbf{q}} C(q)\left[b_{\mathbf{q}} e^{i \mathbf{q} \cdot \mathbf{r}}+b_{\mathbf{q}}^{\dagger} e^{-i \mathbf{q} \cdot \mathbf{r}}\right],
$$

in which $b_{\mathbf{q}}^{\dagger}$ and $b_{\mathbf{q}}$ are the phonon creation and annihilation operators, respectively. The interaction strength $C(q)$ for various types of phonons considered here is given as follows. For polar-optical phonons, the interaction strength reads

$$
|C(q)|^{2}=2 \pi e^{2} \hbar \omega_{0}\left(\frac{1}{\epsilon_{\infty}}-\frac{1}{\epsilon_{0}}\right) \frac{1}{q^{2}}
$$

whereas for acoustic phonons coupled through deformation potential we have

$$
|C(q)|^{2}=\frac{\hbar D^{2}}{2 d v_{s}} q
$$

and for acoustic phonons in the piezoelectric coupling

$$
|C(q)|^{2}=\frac{\hbar K^{2} e^{2} v_{s}}{2 \epsilon_{0}} \frac{1}{q} .
$$

In the above expressions for $C(q)$ 's, $\epsilon_{\infty}$ and $\epsilon_{0}$ are the high-frequency and static dielectric constants of the medium, $D$ and $K$ are the deformation potential and piezoelectric constants, $v_{\boldsymbol{s}}$ is the sound velocity, and $d$ is the mass density.

Expressing the interaction Hamiltonian [Eq. (6)] in cylindrical coordinates is facilitated by the following expression

$$
e^{ \pm i \mathbf{q} \cdot \mathbf{r}}=e^{ \pm \boldsymbol{q}_{z} z}\left[J_{0}\left(q_{\perp} \rho\right)+2 \sum_{n=1}^{\infty}( \pm i)^{n} J_{n}\left(q_{\perp} \rho\right) \cos (n \phi)\right]
$$

where we have used the following notations: $\mathbf{q}=\left(q_{\perp}, q_{z}\right)$ and $\mathbf{r}=(\rho, \phi, z)$. In this work, we are considering only the lowest subband, thus the term involving summation over the index $n$ in Eq. (11) does not contribute to the scattering rate. In systems where higher subbands are also occupied, the above expression can be conveniently employed.

\section{Dielectric function}

The effects of screening have been included by renormalizing the electron-phonon interaction by the dielectric function, viz., $H_{e-\mathrm{ph}} \rightarrow H_{e-\mathrm{ph}} / \varepsilon(q)$. The dielectric function to be employed here should be calculated using the wave functions introduced for the infinite potentialwell model. In a recent work ${ }^{23}$ we have used the RPA dielectric function which was more appropriate for bulk systems, and found a decrease in scattering rates due to the screened electron-phonon interactions. We assume that the density of electrons is such that all the electrons are in the lowest subband and model the Q1D dielectric function as

$$
\varepsilon\left(q_{z}\right)=1+\frac{4}{\epsilon_{0} \pi q_{z} a_{B}} V\left(q_{z}\right) \ln \left|\frac{2 k_{F}+q_{z}}{2 k_{F}-q_{z}}\right|
$$

where $V\left(q_{z}\right)$ is the interaction potential between the electrons occupying the lowest subband, $\epsilon_{0}$ is the dielectric constant of the medium, and $a_{B}$ is the effective Bohr radius. We use the expression given ${ }^{24}$ by

$$
\begin{aligned}
V\left(q_{z}\right)=\frac{36}{\left(q_{z} R\right)^{2}} & {\left[\frac{1}{10}-\frac{2}{3\left(q_{z} R\right)^{2}}+\frac{32}{3\left(q_{z} R\right)^{4}}\right.} \\
& \left.-\frac{64}{\left(q_{z} R\right)^{4}} I_{3}\left(q_{z} R\right) K_{3}\left(q_{z} R\right)\right],
\end{aligned}
$$

where $I_{n}(x)$ and $K_{n}(x)$ are the modified Bessel functions of the first and second kind, respectively. In previous studies, ${ }^{13,22} V\left(q_{z}\right)$ has been evaluated using approximate wave functions. The above form of the dielectric function exhibits a singularity at $q=2 k_{F}$ (Peierls instability), which is a characteristic of $1 \mathrm{D}$ electron systems. This divergence can be circumvented by considering the temperature dependence.

The temperature dependent static dielectric function can be obtained by simply integrating over the chemical potential dependent dielectric function at zero temperature. ${ }^{26}$ We quote the final result for the temperature dependent static dielectric function ${ }^{26}$

$$
\varepsilon_{T}(q)=1+\frac{4}{\epsilon_{0} \pi q_{z} a_{B}} V(q) g\left(q_{z}\right),
$$

where

$$
\begin{array}{r}
g\left(q_{z}\right)=\frac{1}{4 k_{B} T} \int_{0}^{\infty} d \mu^{\prime} \ln \left|\frac{2 \sqrt{2 m \mu^{\prime}}+q_{z}}{2 \sqrt{2 m \mu^{\prime}}-q_{z}}\right| \\
\times \cosh ^{-2}\left(\frac{\mu^{\prime}-E_{F}}{2 k_{B} T}\right),
\end{array}
$$

in which $E_{F}$ is the Fermi energy at $T=0$, and $k_{B}$ is the Boltzmann constant. 


\section{Mobility}

The mobility of carriers confined to moving along the axis of a thin semiconducting wire is

$$
\mu=e \sum_{k_{z}}\left(\frac{\hbar k_{z}}{m}\right)^{2} \tau_{t}\left(k_{z}\right) \frac{\partial f_{0}}{\partial E}\left[\sum_{k_{z}} f_{0}(E)\right]^{-1}
$$

where we have assumed that the SQL conditions are valid, i.e., the carriers are in their lowest quantum states as far as their motion transverse to the axis of the wire is concerned. Here, $f_{0}(E)$ is the finite temperature distribution function for the electrons, which we take to be the Fermi function, with $E$ given by Eq. (2). The above expression for mobility reduces to $\mu=e \tau_{t}\left(k_{F}\right) / m$ in the zero-temperature case.

\section{RESULTS AND DISCUSSION}

We have used material parameters appropriate for GaAs in the numerical calculations we present here. Electron-acoustic-phonon interaction considered here within the deformation potential model is characterized by the deformation potential $D=13.5 \mathrm{eV}$, the mass density of the system $d=5.3 \mathrm{gr} / \mathrm{cm}^{3}$, and the longitudinal sound velocity in GaAs $v_{s}=5.37 \times 10^{5} \mathrm{~cm} / \mathrm{s}$. For acoustic phonons in the piezoelectric coupling the dimensionless piezoelectric constant is given as $K=0.052$. The acoustic-phonon energies here are taken to be $\hbar \omega_{\mathbf{q}}=$ $\hbar v_{s} q$. In the case of electron-optical-phonon interaction, we assume the phonons are dispersionless and have the energy in the bulk $\hbar \omega_{0}=36.2 \mathrm{meV}$. The values of the high-frequency $\epsilon_{\infty}$ and static $\epsilon_{0}$ dielectric constants are taken to be 10.9 and 12.9 , respectively, and we have used the band mass of electrons $m=0.067 m_{e}$ where $m_{e}$ is the bare electron mass. We take the electron density of the GaAs to be $n=1.0 \times 10^{6} \mathrm{~cm}^{-1}$, which corresponds to $E_{F} \approx 14 \mathrm{meV}$ (around zero temperature), and $k_{F}=0.016 \AA^{-1}$. These numbers indicate that only the $n=0$ level will be populated for $R \leq 150 \AA$, making our size-quantum-limit assumption valid (see also Ref. 13 for similar considerations). Also the thermal de Broglie wavelength $\lambda_{T}$ at $T=300 \mathrm{~K}$ is around $300 \AA$.

We first show our results for the electron-opticalphonon interaction. In Fig. 1 the inverse scattering time $\tau^{-1}$ is depicted for two different wire radii, (a) $R=50$ $\AA$ and (b) $R=100 \AA$, as a function of the initial electron energy $E$ in the $z$ direction. The full curves indicate the effects of screened electron-phonon interaction on $\tau^{-1}$, whereas the dashed curves are obtained without screening. The upper and lower curves show emission and absorption rates, respectively. We observe that the screening effects lower the scattering rates in general, and in the case of $R=50 \AA$ wire, this reduction is about $70 \%$. As the wire radius increases, screening effects seem to slightly decrease. For instance, the reduction in the emission and absorption rates becomes about $60 \%$ for $R=100 \AA$. Constantinou and Ridley ${ }^{15}$ have found a reduction in the scattering rates (by about $25 \%$ ) due to the
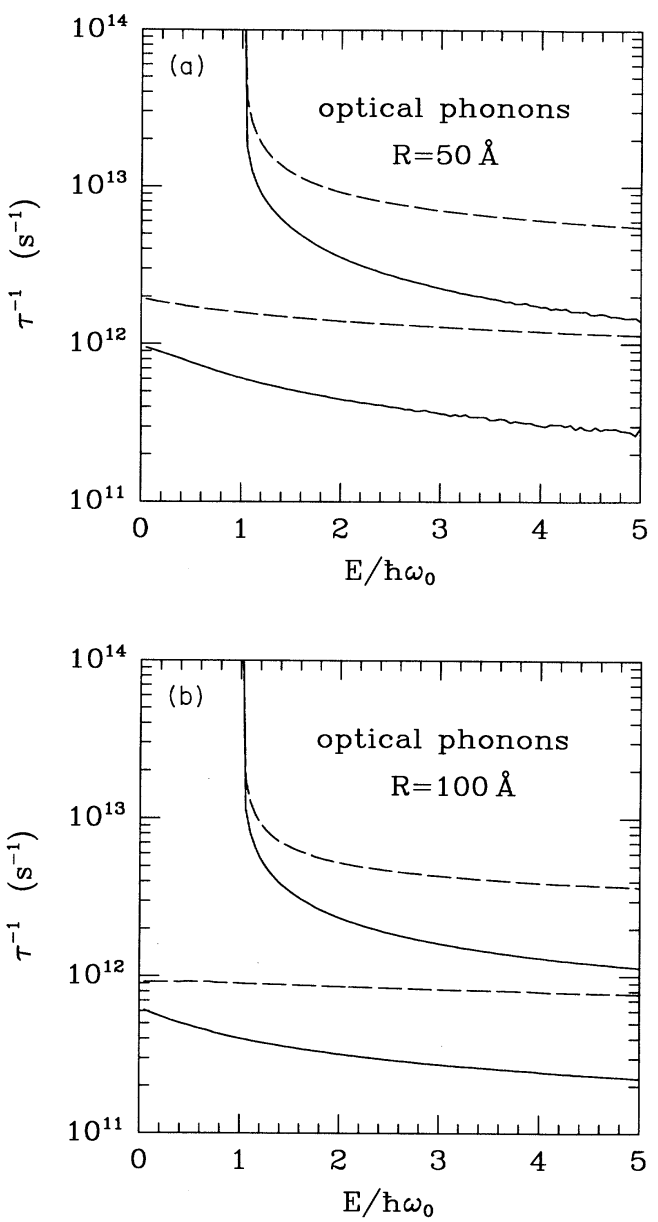

FIG. 1. The inverse scattering time $\tau_{s}^{-1}$ due to electron-optical-phonon interaction for the extreme quantum limit in a quantum-well wire of circular cross section, with radius (a) $R=50 \AA$ and (b) $R=100 \AA$. Solid and dashed curves give $\tau^{-1}$ with and without screening, respectively, for emission (top curves) and absorption (bottom curves) processes at $T=300 \mathrm{~K}$.

inclusion of a finite well height for $R=50 \AA$. Note also the scales in Figs. 1(a) and 1(b) to see the change in the scattering rate $\tau^{-1}$ as the wire radius $R$ varies.

We present our results for the scattering rates due to electron-acoustic-phonon interaction in the deformation potential coupling in Fig. 2. Size dependence of $\tau^{-1}$ (a) $R=50 \AA$ and (b) $R=100 \AA$ is plotted as a function of the initial energy $E$, and we again use the polaroptical-phonon energy $\hbar \omega_{0}$ for scaling purposes. Here the temperature is taken to be $50 \mathrm{~K}$, since the electronacoustic-phonon scattering is known to be dominant in the temperature range $10<T<50 \mathrm{~K}$. The solid and dashed curves indicate the scattering rates with and without dielectric screening, respectively. In contrast to the calculation for electron-optical-phonon scattering, here we resort to the elastic approximation, viz., $E\left(k_{z}\right)$ or $E\left(k_{z}^{\prime}\right) \gg \hbar \omega_{\mathbf{q}}$, and replace the energy conservation expression $\delta\left[E\left(k_{z}\right)-E\left(k_{z}^{\prime}\right) \pm \hbar \omega_{\mathbf{q}}\right]$ with $\delta\left[E\left(k_{z}\right)-E\left(k_{z}^{\prime}\right)\right]$. We observe that the screening effects in the case of 

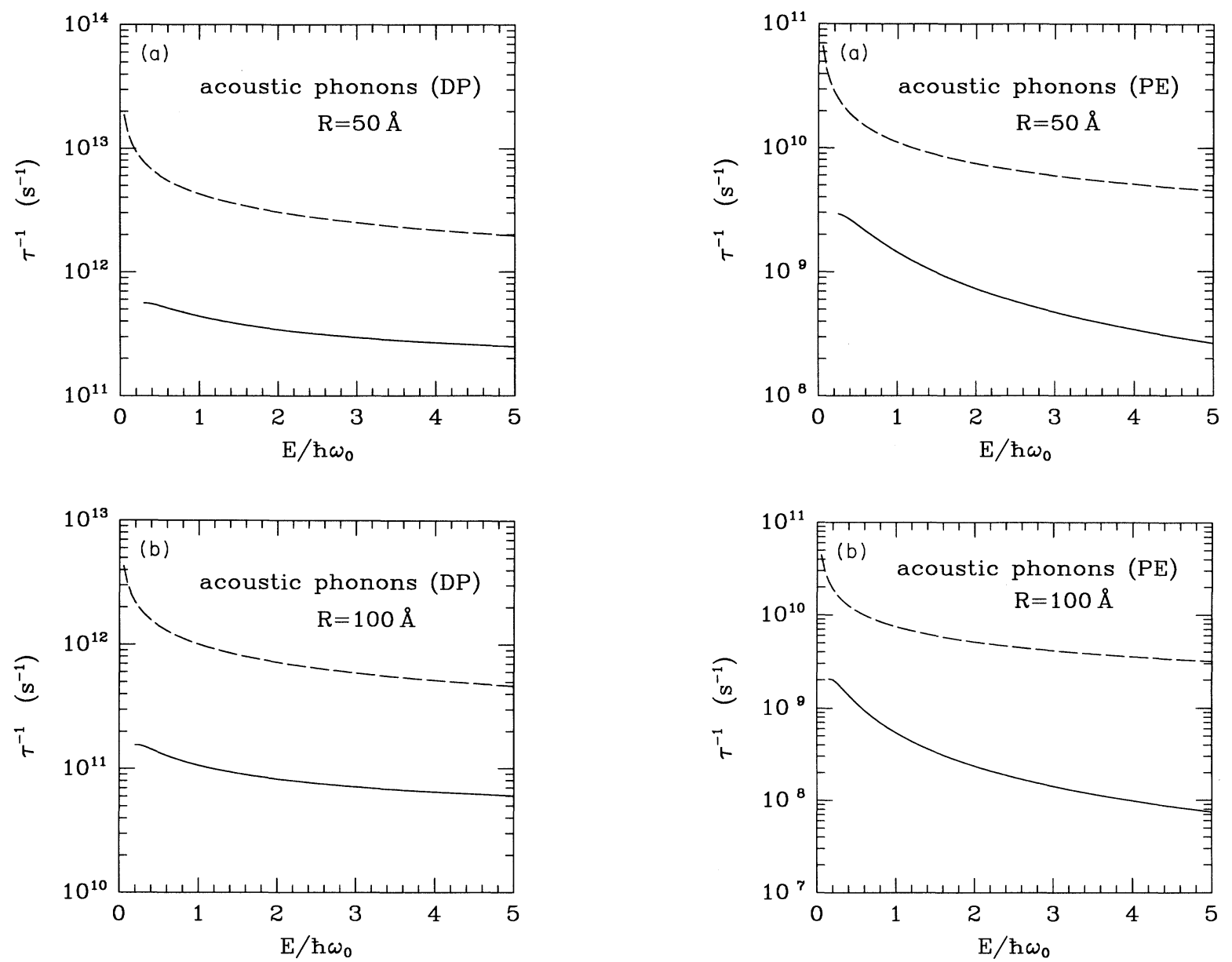

FIG. 2. The inverse scattering time $\tau_{s}^{-1}$ due to electron-acoustic-phonon interaction in the deformation potential coupling for the extreme quantum limit in a quantum-well wire of circular cross section, with radius (a) $R=50 \AA$ and (b) $R=100 \AA$. Solid and dashed curves give $\tau^{-1}$ with and without screening, respectively, for the emission process at $T=50 \mathrm{~K}$.

electron-acoustic-phonon interaction in deformation potential coupling are quite pronounced as compared to the electron-optical-phonon scattering. In fact, for the $R \approx 100 \AA$ radius wire we see an $80 \%$ reduction in the calculated scattering rate. On the other hand, as we shall see below, the relaxation time is not affected by the screening.

In Figs. 3(a) and 3(b), we show the scattering rates due to electron-acoustic-phonon interaction in the piezoelectric coupling. As in Fig. 2, we use $\hbar \omega_{0}$ for scaling purposes and $T=50 \mathrm{~K}$. The solid and dashed curves give the scattering rates with and without dielectric screening, respectively. As in the previous cases, the effects of screened electron-phonon interaction increase somewhat as the wire radius increases. For both the deformation potential and the piezoelectric coupled acoustic phonons, we have only reported the emission rates, since, at the temperature of interest here $(300 \mathrm{~K})$, the phonon occu-

pation factors $N_{\mathbf{q}}$ and $N_{\mathbf{q}}+1$ do not differ much, and the equipartition approximation is valid.

The scattering rate results we have presented here were at $T=300 \mathrm{~K}$ for the electron-optical-phonon interaction, and $T=50 \mathrm{~K}$ for the electron-acoustic-phonon interaction. The relative importance of various electron-phonon interactions at these temperatures may be assessed by the scales of Figs. 1(a)-3(b). It has been known that the dominant phonon scattering mechanism at low temperatures is due to acoustic phonons. At low temperatures, only the emission process takes place, since there are not enough phonons to be absorbed.

It has been $\operatorname{argued}^{8,15}$ that the dimensionality effects are enhanced in 1D structures, and the singularity in the phonon emission rate at $E=\hbar \omega_{0}$ (for electron-opticalphonon interaction) is a consequence of the 1D density of states which is proportional to $\left(E / \hbar \omega_{0} \mp 1\right)^{-1 / 2}$ for emission and absorption processes, respectively. That there 
is no phonon emission below $E=\hbar \omega_{0}$ [see Figs. 1(a) and 1(b)] comes from the fact that we assume the bulk optical phonons are dispersionless, viz., $\omega_{\mathbf{q}}=\omega_{0}$. Similar density of states effects occur for electron-acoustic-phonon emission rates [see Figs. 2(a)-3(b)], but because of the elastic approximation we employ, the onset of phonon emission is shifted to $E=0$. To assess the validity of the elastic approximation $\left[E\left(k_{z}\right)\right.$ or $\left.E\left(k_{z}^{\prime}\right) \gg \hbar \omega_{\mathbf{q}}\right]$, we have also performed the rate calculations for electron-acoustic-phonon interaction without this assumption and found no significant changes at the level of accuracy sought here.

In considering the effects of screening on the electronphonon scattering rates in Q1D quantum-well wires, we have used the dielectric function $\varepsilon(q)$ in the RPA. For GaAs with carrier density $n=1.0 \times 10^{6} \mathrm{~cm}^{-1}$, and assuming the effective mass of electrons to be $m=0.07 m_{e}$, we obtain $r_{s}=6.4$. Although strictly speaking, the RPA is valid only for $r_{s} \ll 1$ (high density limit), we have found that the scattering rates are not altered very much by using, for example, the Hubbard approximation to the dielectric function. In Fig. 4 we show the temperature dependent static dielectric function $\varepsilon(q)$ at $T=300 \mathrm{~K}$ (indicated by the solid line), in comparison to the zerotemperature $\varepsilon(q)$ (indicated by the dotted line) in the RPA. Evidently, the temperature dependence modifies $\varepsilon(q)$ at small $q$, which, in turn, is quite different from the noninteracting dielectric function in the same region. Also shown (dashed line) is the temperature dependent $\varepsilon_{T}(q)$ calculated with the approximate $V\left(q_{z}\right),{ }^{13,22}$ and it lies between the zero-temperature and present results. Examining the various forms of the electron-phonon interaction parameter $C(q)$ [Eqs. (8)-(10)] and considering their contribution to the scattering time $\tau$, we expect the effects of screened interaction to be largest for the electron-optical-phonon scattering and smallest for the electron-acoustic-phonon scattering (deformation potential). We have accounted for the temperature dependence of the dielectric function using the formulation ${ }^{26}$ given in

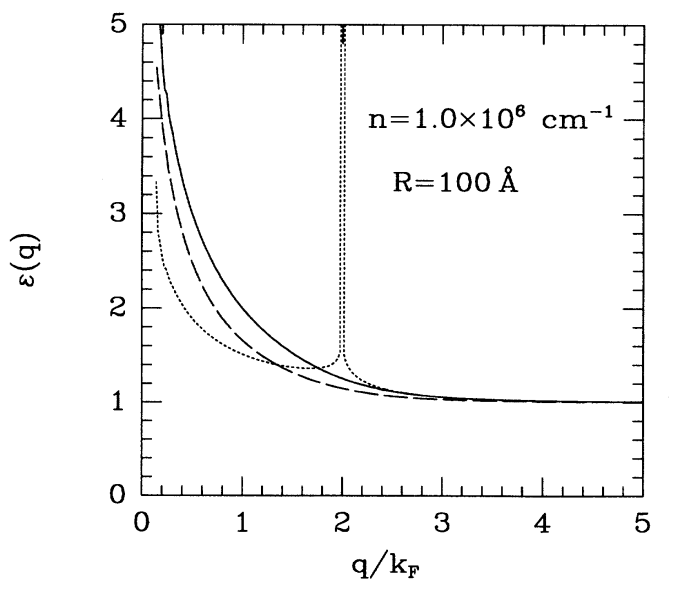

FIG. 4. The temperature dependent dielectric function used in the screened electron-phonon interactions. Solid line, $\varepsilon_{T}(q)$ (this work); dashed line, $\varepsilon_{T}(q)$ of Ref. 22, for both $T=300 \mathrm{~K}$ and $R=100 \AA$, and dotted line, $\varepsilon(q)$ at $T=0$. The number density of electrons is $n=1.0 \times 10^{6} \mathrm{~cm}^{-1}$. the preceding section. This has been crucial $^{13}$ in softening the divergence at $q=2 k_{F}$ in $1 \mathrm{D}$ dielectric function. Indeed, we have found that the temperature dependence of the dielectric function has the effect of lowering the scattering rates. It is clear from Fig. 4 that the screening effects would be more significant at low temperatures than high temperatures, as noted by other researchers.

The effects of electron-acoustic-phonon interaction on the mobility of Q1D electron systems attracted some interest. ${ }^{27}$ It was found that the screening plays no effect in the temperature dependent mobility due to the acoustic-phonon scattering in the deformation potential coupling. We display in Fig. 5 the inverse relaxation time $\tau_{t}$ for electron-acoustic phonon interaction in the deformation potential coupling, for a $R=50 \AA$ quantum well wire. The relaxation time $\tau_{t}$ different in principle than the scattering time $\tau_{s}$ enters the mobility calculation, and our results indicate that the screening should have very little effect, except for small energies. This is consistent with the earlier findings. ${ }^{27}$

Constantinou and Ridley $^{15}$ have found $\sim 25 \%$ reduction in the scattering rates due to the finite depth of the confining quantum well in Q1D semiconductor structures. Our investigation shows that a similar order of magnitude effect can be accounted for by the screened electron-phonon interactions. Therefore, if the screened electron-phonon interactions are used with a finite quantum-well model of confinement, further reduction in the scattering rates is possible. Lowering in the scattering rates due to screened electron-phonon interactions made explicit in our calculations have been previously anticipated. ${ }^{8,28}$ Although we have presented our results only for GaAs, with the appropriate choice of the material parameters, different substances such as InAs, InSb, CdS, etc., can also be studied. Our numerical calculations show that the lowering of the scattering rates due to screened electron-phonon interactions would en-

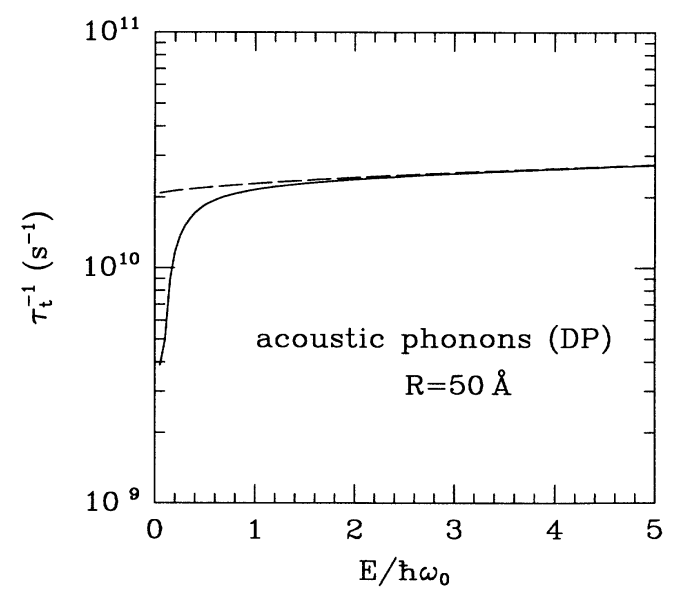

FIG. 5. The inverse relaxation time $\tau_{t}^{-1}$ due to electron-acoustic-phonon interaction in the deformation potential coupling for the extreme quantum limit in a quantum-well wire of circular cross section, with radius $R=50 \AA$. Solid and dashed curves give $\tau^{-1}$ with and without screening, respectively, for the emission process at $T=50 \mathrm{~K}$. 
hance the mobility in Q1D samples which will have important technological implications in terms of device applications.

We have studied the size effects on the screened electron-phonon interactions in Q1D quantum-well wires by calculating the scattering rates. We have considered the interaction of electrons with polar-optical and acoustic phonons. Acoustic phonons, in turn, are treated in the deformation potential and piezoelectric couplings. Screening effects are introduced by renormalizing the electron-phonon interactions with the temperature dependent dielectric function $\varepsilon_{T}(q)$. Effects of screened electron-phonon interactions on the single-particle scattering rates are strongest in the case of electron-optical- phonon scattering. In this work we have assumed the bulk-phonon interactions, which is valid for the large quantum-well wire radius, i.e., $R>50 \AA$. For wire radii, $R<50 \AA$, it will be necessary to consider the confined phonon modes.

\section{ACKNOWLEDGMENTS}

This work was supported by the Scientific and Technical Research Council of Turkey (TUBITAK) under Grant No. TBAG-1155. Useful discussions with Professor M. Tomak and Professor A. Erçelebi are gratefully acknowledged.
${ }^{1}$ H. Sakaki, Jpn. J. Appl. Phys. 19, L735 (1980).

${ }^{2}$ P. M. Petroff, A. C. Gossard, R. A. Logan, and W. Wiegmann, Appl. Phys. Lett. 41, 635 (1982).

3 T. J. Thornton, M. Pepper, H. Ahmed, D. Andrews, and G. J. Davies, Phys. Rev. Lett. 56, 1198 (1986).

${ }^{4}$ J. Cibert, P. M. Petroff, G. J. Dolan, S. J. Pearton, A. C. Gossard, and J. H. English, Appl. Phys. Lett. 49, 1275 (1986).

${ }^{5}$ H. Temkin, G. J. Dolan, M. B. Parish, and S. N. G. Chu, Appl. Phys. Lett. 40, 413 (1987).

${ }^{6}$ V. Arora, Phys. Rev. B 23, 5611 (1981); Phys. Status Solidi B 105, 707 (1981).

${ }^{7}$ F. A. Riddock and B. K. Ridley, Surf. Sci. 142, 260 (1982).

${ }^{8}$ J. P. Leburton, J. Appl. Phys. 56, 2850 (1984).

${ }^{9}$ S. Briggs and J. P. Leburton, Phys. Rev. B 38, 8163 (1988).

${ }^{10}$ G. J. Iafrate, D. K. Kerry, and R. K. Reich, Surf. Sci. 113, 485 (1982).

${ }^{11}$ S. Briggs, B. A. Mason, and J. P. Leburton, Phys. Rev. B 40, 12001 (1989).

12 J. Lee and H. N. Spector, J. Appl. Phys. 54, 3921 (1983).

${ }^{13}$ G. Fishman, Phys. Rev. B 34, 2394 (1986); 36, 7448 (1987).

${ }^{14}$ G. Bastard, J. A. Brum, and R. Ferreira, Solid State Phys. 44, 229 (1991).
${ }^{15}$ N. C. Constantinou and B. K. Ridley, J. Phys. Condens. Matter 1, 2283 (1989).

${ }^{16}$ D. Jovanovic, S. Briggs, and J. P. Leburton, Phys. Rev. B 42, 11108 (1990).

${ }^{17}$ J. P. Leburton and D. Jovanovic, Semicond. Sci. Technol. 7, B202 (1992).

${ }^{18}$ V. B. Campos, M. H. Degani, and O. Hipólito, Solid State Commun. 79, 473 (1991).

${ }^{19}$ P. K. Basu and B. R. Nag, J. Phys. C 14, 1519 (1981).

${ }^{20}$ J. Lee and M. O. Vassell, J. Phys. C 17, 2525 (1984).

${ }^{21}$ W. S. Li, S.-W. Gu, T. C. Au-Yeung, and Y. Y. Yeung, Phys. Lett. A 166, 377 (1992).

${ }^{22}$ J. Lee and H. N. Spector, J. Appl. Phys. 57, 366 (1985).

${ }^{23}$ B. Tanatar, J. Phys. Condens. Matter 5, 2203 (1993).

${ }^{24}$ A. Gold and A. Ghazali, Phys. Rev. B 41, 7626 (1990).

${ }^{25}$ H. Sakaki, J. Vac. Sci. Technol. 19, 148 (1981).

${ }^{26}$ P. Maldague, Surf. Sci. 73, 296 (1978); N. R. Arista and W. Brandt, Phys. Rev. A 29, 1471 (1984).

${ }^{27}$ W. Walukiewicz, Phys. Rev. B 37, 8530 (1988); C. E. Leal, I. C. da Cunha Lima, E. A. de Andrada e Silva, and A. Troper, ibid. 38, 3525 (1988).

${ }^{28}$ J. Shah, A. Pinczuk, H. L. Störmer, A. C. Gossard, and W. Wiegmann, Appl. Phys. Lett. 42, 55 (1983). 